\title{
Atom-atom entanglement by single-photon detection
}

\author{
L. Slodička ${ }^{1}$, G. Hétet ${ }^{1}$, N. Röck ${ }^{1}$, P. Schindler ${ }^{1}$, M. Hennrich ${ }^{1}$, and R. Blatt ${ }^{1,2}$ \\ 1 Institut für Experimentalphysik, Universität Innsbruck, Technikerstraße 25, 6020 Innsbruck, \\ Austria $^{2}$ Institut für Quantenoptik und Quanteninformation der Österreichischen \\ Akademie der Wissenschaften, Technikerstraße 21a, 6020 Innsbruck, Austria
}

(Dated: November 1, 2018)

\begin{abstract}
A scheme for entangling distant atoms is realized, as proposed in the seminal paper by Cabrillo et al. [Phys. Rev. A 59, 1025 (1999)]. The protocol is based on quantum interference and detection of a single photon scattered from two effectively one meter distant laser-cooled and trapped atomic ions. The detection of a single photon heralds entanglement of two internal states of the trapped ions with high rate and with a fidelity limited mostly by atomic motion. Control of the entangled state phase is demonstrated by changing the path length of the single-photon interferometer.
\end{abstract}

PACS numbers:

The generation of entanglement between distant physical systems is an essential primitive for quantum communication networks [1, 2] and further tests of quantum mechanics. The realization of heralded entanglement between distant atomic ensembles 3, 4, was amongst the first major achievements in this direction. Probabilistic generation of heralded entanglement between single atoms 8 was demonstrated using single trapped ions [6] and neutral atoms [7] with an entanglement generation rate given by the probability of coincident detection of the two photons coming from the atoms 8,9 . More recently, single neutral atoms trapped at distant locations were entangled by first generating the single atom-photon entanglement and then mapping the photonic state on the electronic state of the second atom [10. A heralding mechanism will however be essential for efficient entanglement and scalability of quantum networks using realistic channels 2, and single qubit operations are required for distributed quantum information processing schemes 11. In this Letter we report on the realization of a fundamental process which fulfills both these conditions by showing entanglement between two well-defined atomic qubits via emission and detection of a single light quanta [6. In this scheme, both the energy and the phase of the emitted single photon are used for entanglement generation. In addition, this mechanism allows the demonstration of a large speedup in entanglement generation rate compared to the previously realized heralded entanglement protocol with single atoms [6, 8, This result will enable the practical distribution of quantum information over long distances using single atom architectures.

Entanglement of distant single atoms through the detection of a single photon, as proposed in the seminal work of Cabrillo et al. [6], is both a fundamental and a promising technique for the field of quantum information. The interconnection between quantum nodes based on this scheme would provide efficient distribution of quantum information in large scale quantum networks [8, 9]. To generate heralded entanglement, two atoms $(A, B)$ are both prepared in the same long-lived electronic state $|g g\rangle$. Each atom is excited with a small probability $p_{e}$ to another metastable state $|e\rangle$ through a

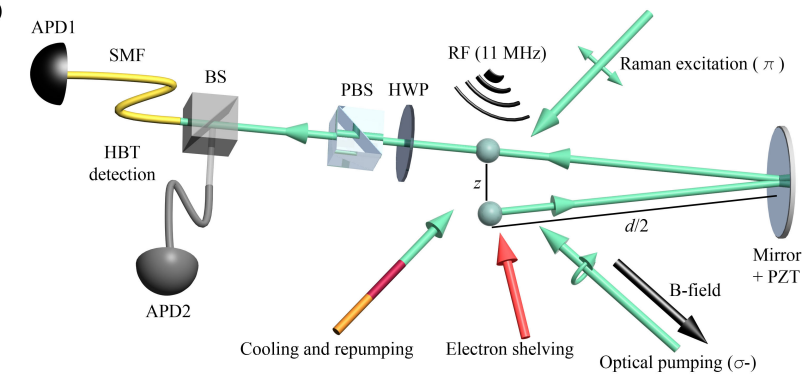

b)

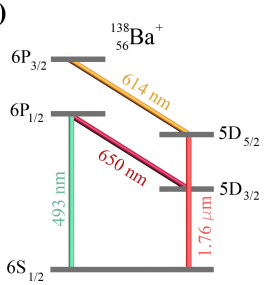

c)

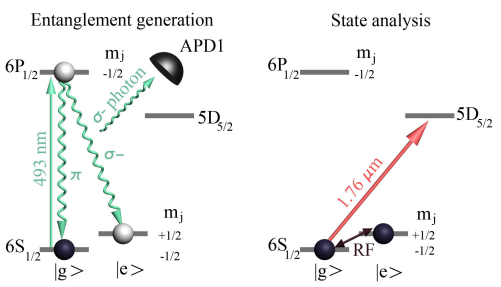

FIG. 1: Experimental procedure for entanglement generation. a) The fluorescence of the two ions is overlapped using a distant mirror which sets the effective distance between them to $d=1$ meter. A half wave plate (HWP), a polarizing beam splitter (PBS) and a single-mode optical fiber select the polarization and the spatial mode before an avalanche photodiode (APD1). A non-polarizing beam-splitter and an additional avalanche photodiode (APD2) can be inserted to form a Hanbury-Brown-Twiss setup. See details in main text. b) Level scheme of ${ }^{138} \mathrm{Ba}^{+}$including the wavelengths of the lasers used in our experiment. c) Experimental sequence. Spontaneous Raman scattering to $|e\rangle$ triggers emission of a single photon from the two atoms. Upon successful detection of a $\sigma^{-}$ photon, state analysis comprising coherent radio-frequency (RF) pulses at $11 \mathrm{MHz}$, and electron shelving to the $5 \mathrm{D}_{5 / 2}$ level are performed. See details in main text.

spontaneous Raman process $(|g\rangle \rightarrow|i\rangle \rightarrow|e\rangle)$ by weak excitation of the $|g\rangle \rightarrow|i\rangle$ transition and spontaneous emission of the single photon on the $|i\rangle \rightarrow|e\rangle$. Here $|i\rangle$ denotes an auxiliary atomic state with short lifetime. This Raman process entangles each of the atom's internal states with the emitted photon number, so the state 
of each atom and its corresponding light mode can be written as $\sqrt{1-p_{e}}|g, 0\rangle e^{i \phi_{L}}+\sqrt{p_{e}}|e, 1\rangle e^{i \phi_{D}}$. Here, the phases $\phi_{L}$ and $\phi_{D}$ correspond to the phase of the exciting laser at the position of atom $A$ and the phase acquired by the spontaneously emitted photon on its way to the detector, respectively. Indistinguishability of the photons from the two atoms is achieved by overlapping their corresponding modes, for example using a beam splitter. The total state of the system consisting of both atoms and the light modes is then $\left(1-p_{e}\right) e^{i\left(\phi_{L, A}+\phi_{L, B}\right)}|g g, 0\rangle+$ $\sqrt{p_{e}\left(1-p_{e}\right)}\left(e^{i\left(\phi_{L, A}+\phi_{D, B}\right)}|e g, 1\rangle+e^{i\left(\phi_{L, B}+\phi_{D, A}\right)}|g e, 1\rangle\right)+$ $p_{e} e^{i\left(\phi_{D}, A+\phi_{D}, B\right)}|e e, 2\rangle$. Single photon detection projects the two-atom state onto an entangled state $\left|\Psi^{\phi}\right\rangle=$ $\frac{1}{\sqrt{2}}\left(|e g\rangle+e^{i \phi}|g e\rangle\right)$. Since at least one atom must be excited, the probability of measuring such a state is then $1-p_{e}^{2}$. Here $p_{e}^{2}$ is the probability of simultaneous excitation of both atoms. The absolute success probability of the entangled state generation in one experimental run is then $P_{\text {succ }}=2 p_{e}\left(1-p_{e}\right) \eta$, where $\eta$ is the overall detection efficiency of the generated photons. The phase of the generated entangled state $\phi$ corresponds to the sum of the phase difference acquired by exciting beam at the position of the two atoms and the phase difference acquired by the photons from the respective atoms upon travelling to the detector. The only limiting factor here is the probability of simultaneous excitation of the two atoms $p_{e}^{2}$, which can be, in principle, made arbitrarily small.

To experimentally demonstrate the creation of such a single-photon heralded entanglement two barium ions are trapped and cooled in a linear Paul trap 4. As shown in Fig. 1 a) and b), laser light at $493 \mathrm{~nm}$ is used to Doppler-cool the ions and to detect their electronic states by means of electron shelving, and a laser field at $650 \mathrm{~nm}$ repumps the atoms to the $P_{1 / 2}$ level from the metastable $\mathrm{D}_{3 / 2}$ state. By carefully adjusting the cooling and trapping parameters, the ions are always well within the Lamb-Dicke limit so that the photon recoil during the Raman scattering process is mostly carried by the trap. This ensures that only minimal information is retained in the motion of the ion about which atom scattered the photon during the entanglement generation process. The fluorescence photons are efficiently collected by two high numerical aperture lenses $(\mathrm{NA} \approx 0.4$ ) placed $14 \mathrm{~mm}$ away from the atoms. A magnetic field of $0.4 \mathrm{mT}$ is applied at an angle of 45 degrees with respect to the two-ion axis and defines the quantization axis. After passing through a polarizing beam splitter that blocks the $\pi$-polarized light and lets $\sigma$-polarized light pass, the spatial overlap of the photons is guaranteed by collecting the atomic fluorescence of the first ion in a single mode optical fiber, whilst the fluorescence of the second ion is sent to a distant mirror that retro-reflects it in the same optical fiber [14. The fluorescence of the two ions (including the Raman scattered light) is then detected by an avalanche photodiode with a quantum efficiency of $60 \%$.

For efficient generation of the two-atom entangled state, the emitted photons must be indistinguishable in all degrees of freedom at the position of the triggering detector. We characterize their indistinguishability by a measurement of the first and second order correlation functions (see supplemental material A). These measurements yield unambiguous separation between the major decoherence mechanisms and lead to the conclusion that which-way information given by atomic motion is the main source of distinguishability.

In the entanglement generation procedure, we first Doppler-cool the ions and stabilize the mirror-ion distance $d / 2$ by locking the position of the interference fringe measured during the Doppler cooling sequence to a chosen position, see Fig 1 - a). The ion internal states are then prepared to the Zeeman substates $\left|6 S_{1 / 2}, m_{j}=-1 / 2\right\rangle=$ $|g\rangle$ by optical pumping with a circularly polarized laser pulse propagating along the magnetic field. Then, a weak horizontally polarized laser pulse (Raman excitation) excites both ions on the $\mathrm{S}_{1 / 2} \leftrightarrow \mathrm{P}_{1 / 2}$ transition with a probability $p_{e}=0.07$ through a resonant spontaneous Raman scattering to the other Zeeman sublevel $\left(\mathrm{m}_{j}=+1 / 2\right)$ of the $6 \mathrm{~S}_{1 / 2}$ state, $|e\rangle$. The electronic state of each ion is at this point entangled with the number of photons $|0\rangle$ or $|1\rangle$ in the $\sigma^{-}$polarized photonic mode. Provided that high indistinguishability of the two photonic channels is assured and that simultaneous excitation of both atoms is negligible, detection of a single $\sigma^{-}$photon on the APD projects the two-ion state onto the maximally entangled state $|\Psi\rangle=\frac{1}{\sqrt{2}}\left(|g e\rangle+|e g\rangle e^{i k d}\right)$, where $k$ is the wavenumber of the $493 \mathrm{~nm}$ fluorescence. The phase factor $e^{i k d}$ corresponds here solely to the phase difference $\phi_{D, A}-\phi_{D, B}$ acquired by the emitted photon upon its way to the detector. The phase difference of the excitation laser at the position of two ions $\phi_{L, A}-\phi_{L, B}$ is fixed to $n \times 2 \pi, n \in I$ by setting the mutual distance between the ions in the trap to $z=n \lambda / \cos \theta$, where $\theta$ is the angle between the Raman-excitation laser direction and the ion-crystal axis. We will first demonstrate a successful preparation of the Bell state $\left|\Psi^{+}\right\rangle$for the phase $e^{i k d}=1$ corresponding to an antinode of the interference fringe.

Following the detection of a Raman scattered $\sigma^{-}$photon, we coherently manipulate the generated two-atom state to allow for measurements in a different bases. As shown in Fig 1 $\mathrm{c}$ ), this is done by first applying radiofrequency (RF) pulses that are resonant with the $|g\rangle \leftrightarrow$ $|e\rangle$ transition of both atoms. Discrimination between the two Zeeman sub-levels of the $\mathrm{S}_{1 / 2}$ state is finally done by shelving the population of the $\mathrm{m}_{j}=-1 / 2$ state to the metastable $\mathrm{D}_{5 / 2}$ level using a narrowband $1.76 \mu \mathrm{m}$ laser [4. The fluorescence on the $\mathrm{S}_{1 / 2} \leftrightarrow \mathrm{P}_{1 / 2}$ transition allows us to measure the two-atom state. By setting the appropriate thresholds on the fluorescence counting histogram, we can discriminate the three possible cases where no excitations are present in the two atoms, a single excitation is shared between the two atoms, and where two atoms are excited. These events can all be separated with $98 \%$ probability, enabling us to efficiently reconstruct the relevant parts of the density matrix of the two-atom state. The $614 \mathrm{~nm}$ laser field then resets the ions to the $6 \mathrm{~S}_{1 / 2}$ 
state and the same experiment is repeated 100 times.

Fig. 2-a) shows the measurement results obtained without the RF analysis pulses. The results tell us that $89 \pm 3 \%$ of all the triggering events signal that only one of the atoms was excited to the $|e\rangle$ state. The remaining $10 \%$ errors are caused by APD dark counts and double excitation of the ions. Our detection process using a single photomultiplier doesn't allow us to resolve individual $\rho_{e g}$ and $\rho_{g e}$ populations directly, but it tells us the number of the excited atoms, so the sum of these terms. Although individual populations of the $\rho_{e g}$ and $\rho_{g e}$ states are not needed for estimation of the fidelity with the state $\left|\Psi^{+}\right\rangle$, we also experimentally prove that $\rho_{e g}$ and $\rho_{g e}$ populations are approximately the same and depend only on the overall fluorescence detection efficiencies from the two ions. In order to measure the quantum coherence of the generated state, we then apply two consecutive global RF-pulses, each corresponding to the rotation $\hat{R}(\theta, \phi)=\exp \left(-i \frac{\theta}{2}\left(\cos \phi \hat{S}_{x}+\sin \phi \hat{S}_{y}\right)\right)$, where $\hat{S}_{x, y}=\hat{\sigma}_{x, y}^{(1)} \otimes \hat{\sigma}_{x, y}^{(2)}$ is the global Pauli operator acting on both ions. The rotation angle $\theta$ and rotation axis $\phi$ on the Bloch sphere are determined by the duration and the phase of the RF pulses, respectively. We first apply the pulse $\hat{R}(\pi / 2, \pi / 2)$ which performs the unitary rotation $\hat{R}(\pi / 2, \pi / 2)\left|\Psi^{+}\right\rangle \rightarrow\left|\Phi^{-}\right\rangle$, where $\left|\Phi^{-}\right\rangle=\frac{1}{\sqrt{2}}(|g g\rangle-|e e\rangle)$. A second RF-pulse with same duration but with a phase $\phi$ then performs the rotation $\hat{R}(\pi / 2, \phi)\left|\Phi^{-}\right\rangle$. After shelving the state $|e\rangle$ to the metastable level $D_{5 / 2}$, we scatter light from both ions on the cooling transition. From the measured fluorescence rate at different phases $\phi$, we extract the mean value of the parity operator defined as $\hat{P}=\hat{p}_{g g}+\hat{p}_{e e}-\hat{p}_{e g}-\hat{p}_{g e}$, where $\hat{p}_{i j}$ are the projection operators on states $|i j\rangle, i, j \in\{g, e\}[5]$ (see supplemental materials B).

Fig. 2-b), trace (ii), shows the results of the parity operator measurements preceded by two global $\mathrm{RF}$ rotations $\hat{R}(\pi / 2, \phi) \hat{R}(\pi / 2, \pi / 2)$. The measured parity clearly oscillates as a function of phase $\phi$ with contrast of $58.0 \pm 2.5 \%$ and period of $\pi$, a proof that we indeed succeed in preparing an entangled two-ion state close to $\left|\Psi^{+}\right\rangle$[5]. The mean value of the parity operator at zero phase $\langle\hat{P}\rangle_{\phi \rightarrow 0}$ corresponds to the difference between the inner parts and outer-most coherence terms of the density matrix. We evaluate it to be $2 \operatorname{Re}\left(\rho_{\text {ge,eg }}-\rho_{\text {gg,ee }}\right)=$ $0.38 \pm 0.03$. To precisely quantify the fidelity of our state with $\left|\Psi^{+}\right\rangle$, we however need to estimate the real part of the coherence $\rho_{g e, e g}$ itself. This is done by measuring the parity without the first RF rotation. Trace (i) of Fig. 2-b) shows the expectation value of the parity as a function of the phase $\phi$ of the single RF-pulse $\hat{R}(\pi / 2, \phi)$. The only oscillatory term contributing to this parity measurement reads $2\left(\sin (2 \phi) \operatorname{Im} \rho_{\mathrm{gg}, \mathrm{ee}}-\cos (2 \phi) \operatorname{Re} \rho_{\mathrm{gg}, \mathrm{ee}}\right)$. The measured data however shows independence of the parity signal with respect to the phase $\phi$ within the measurement error. Therefore, only the coherence corresponding to the state $\left|\Psi^{+}\right\rangle$contributes to the parity signal (ii). The value of the coherence $\operatorname{Re}\left(\rho_{\mathrm{gg}, \mathrm{ee}}\right)$ estimated from these a)

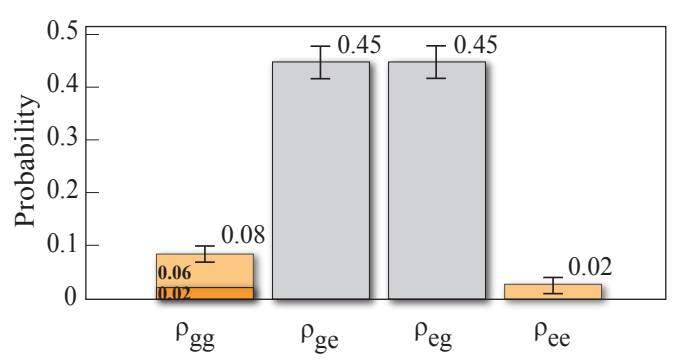

b)

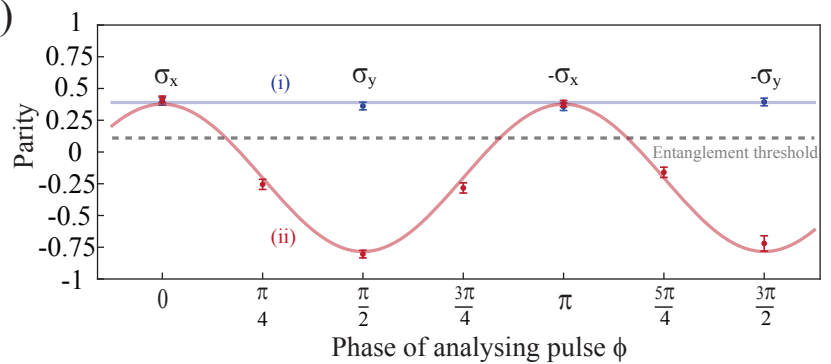

c)

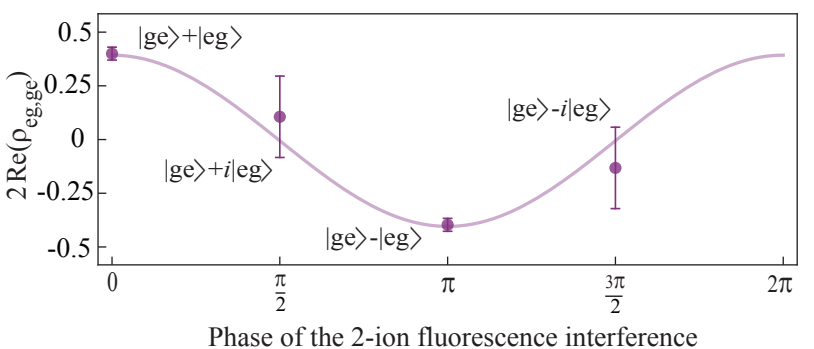

FIG. 2: Characterization of the entangled state. a) Two-atom state populations after the detection of a $\sigma^{-}$photon showing that the total probability of measuring the state with a single excitation is $90 \%$. b) Parity measurements as a function of the RF-phase. Trace (ii) corresponds to the measurement of the atomic populations after two global rotations $\hat{R}^{g}(\pi / 2, \phi) \hat{R}^{g}(\pi / 2, \pi / 2)$. In the measurement of trace (i) only a single global RF-pulse $\hat{R}^{g}(\pi / 2, \phi)$ is applied. The dashed line shows the threshold for entanglement, estimated from the measured diagonal terms. c) Real part of the coherence between the $|g e\rangle$ and $|e g\rangle$ states as a function of the phase of the optical path difference between the two ions.

measurements is $\operatorname{Re}\left(\rho_{\mathrm{gg}, \mathrm{ee}}\right)=0.00 \pm 0.03$. We finally estimate that the fidelity of the generated state with the maximally entangled state $\left|\Psi^{+}\right\rangle$is $F=64 \pm 2 \%$. The threshold for an entanglement is thus surpassed by more than six standard deviations.

The coherence between the $|g e\rangle$ and $|e g\rangle$ states of $38 \pm 3 \%$ is limited by three main processes. First, imperfect populations of $|g e\rangle$ and $|e g\rangle$ states set a limit of $89 \%$ [16. Around $4 \%$ of the coherence loss can be attributed to the finite coherence time of the individual atomic qubits $(120 \mu \mathrm{s})$ due to collective magnetic field fluctuations. Although the generated $\left|\Psi^{+}\right\rangle$state is intrinsically insensitive against collective dephasing [17, 18, a loss of coherence is indeed expected after the rotation of $\left|\Psi^{+}\right\rangle$out of the decoherence-free subspace. The high- 
est contribution to the coherence loss can be attributed to atomic motion, which can provide information about which atom emitted the photon. Around $55 \%$ of the coherence is lost due to the atomic recoil kicks during the Raman scattering (see supplemental material C). Errorbars in the presented measurements results correspond to one standard deviation and are estimated statistically from several experimental runs each giving approximately 120 measurement outcomes. Up to $60 \%$ of the measurement error is caused by the quantum projection noise. Additional uncertainty comes from slow magnetic field drifts with a magnitude of several tens of nT making the RF-driving off-resonant by tens of $\mathrm{kHz}$.

An intrinsic feature of the realized entangling protocol is the dependence of the generated entangled state phase on the optical path difference between the ions. To demonstrate this, we measure the real part of the coherence between the $|g e\rangle$ and $|e g\rangle$ states as a function of the phase factor $k d$. Fig. 2-c) reveals a large change of the real part of the coherence from positive to negative values when going from the maximum to the minimum of the interference signal, in agreement with the $e^{i k d}$ phase dependence of the entangled state.

An important feature of the single-photon heralding mechanism is the high entanglement generation rate that can be achieved. With our experimental set-up, the single photon detection scheme indeed yields a higher rate compared to the two photon scheme proposed by Simon et al. 7, 8, 8, 19. The probability of preparing an entangled state depends on the probability of the single photon detection and the Raman scattering probabilities [8], which in our case gives a total of $P_{\text {succ }}=1.1 \times 10^{-4}$ for each trial run. With an experimental duty cycle of $2.3 \mathrm{kHz}$, this corresponds to 15.4 successful entanglement generation events/minute, which is in good agreement with the experimentally observed $14 \pm 2$ events/minute. A detailed analysis of the overall photon-detection efficiency can be found in the supplemental material E.

We have demonstrated a fundamentally new protocol for generating heralded entanglement between two ions. This was achieved via the scheme proposed in the seminal work of Cabrillo et al. 6] where two atoms are entangled with the emission and detection of only one photon. Such a single-photon scheme allowed us to reach a rate of entanglement generation of 14 events/minute, more than two orders of magnitude higher than the rate obtainable with protocols relying on a two-photon coincidence events with our experimental parameters. The maximally entangled state $\left|\Psi^{+}\right\rangle$is produced with a fidelity of $63.5 \%$ limited mostly by residual atomic motion. These results can be improved by cooling all of the involved motional modes close to their ground state [4] or choosing a different excitation direction to minimize residual which-way information. These improvements, together with the experimental results presented, will enable efficient creation and distribution of entanglement between distant sites with well-defined and controllable atomic qubits. Such entanglement generation corresponds to an essential building block of scalable quantum communication [11] and distributed quantum computation [20 22 architectures with single atoms.

We thank J. Eschner, T. Monz, T. Northup and A. Stute for helpful discussions. This work was supported by the Austrian Science Fund (FWF), the Institut für Quanteninformation $\mathrm{GmbH}$, and a Marie Curie International Incoming Fellowship within the 8th European Framework Program.
[1] Briegel, H. J. et al. Phys. Rev. Lett. 81, 59325935 (1998).

[2] Duan, L. M. et al. Nature 414, 413-418 (2001).

[3] Chou, C. W. et al. Nature 438, 828 (2005).

[4] Chou, C. W. et al. Science 316, 1316 (2007).

[5] Simon, C. \& Irvine, W. T. M. Phys. Rev. Lett. 91, 110405 (2003).

[6] Moehring, D. L. et al. Nature 449, 68-71 (2007).

[7] Hofmann, J. et al. Science 337, 72-75 (2012).

[8] Zippilli, S. et al. New Journal of Physics 10, 103003 (2008).

[9] Luo, L. et al. Fortschritte der Physik 57, 1133-1152 (2009).

[10] Ritter, S. et al. Nature 484, 195200 (2012).

[11] Duan, L. -M. \& Monroe, C. Rev. Mod. Phys. 82, 12091224 (2010).

[12] Cabrillo, C., Cirac, J. I., Garcia-Fernández, P. \& Zoller, P. Phys. Rev. A 59, 1025-1033 (1999).

[13] Slodička, L. et al. Phys. Rev. A 85, 043401 (2012).

[14] We note that in our setup, super/sub-radiance effects that might occur here are on the order of $1 \%$, and can thus be neglected. See Rist, S. et al. Phys. Rev. A 78, 013808 (2008).
[15] Sackett, C. A. et al. Nature 404, 256-258 (2000).

[16] Shirokov, M. et al. International Journal of Theoretical Physics 45, 141-151 (2006).

[17] Roos, C. F. et al. Phys. Rev. Lett. 92, 220402 (2004).

[18] Kielpinski, D. et al. Science 291, 1013-1015 (2001).

[19] Matsukevich, D. N. et al. Phys. Rev. Lett. 100, 150404 (2008).

[20] Jiang, L. et al. Phys. Rev. A 76, 062323 (2007).

[21] Cirac, J. I. et al. Phys. Rev. A 59, 4249-4254 (1999).

[22] Gottesman, D. \& Chuang, I. L. Nature 402, 390-393 (1999). 


\section{Supplemental material}

\section{A. Indistinguishability measurements}

We estimate the degree of indistinguishability of photons emitted by the two atoms by a measurement of the first and second order correlation functions. Fig. 3 3 a), trace (i) shows the second-order correlation function of the scattered light measured using the Hanbury-BrownTwiss detection setup. At zero time delay between two consecutive clicks, $g^{(2)}(\tau=0)=0.98 \pm 0.07$ close to the theoretical value 1 , a signature of high indistinguishability of the spatial and polarization degrees of freedom in this optical mode (see supplemental material D). In order to estimate the amount of motion induced which-way information, we measure the first order correlation function. Fig. 3-b), trace (i) shows the fluorescence intensity as a function of the distance $d$ between the two ions in a regime where elastic scattering dominates. For comparison, trace (ii) shows the interference of the fluorescence of one ion with itself under the same cooling conditions. A contrast of up to $40 \%$ is observed for the interference of the elastic light scattered by the two ions. Temporal decoherence caused by the photon emission and absorption recoils 1 14 is high enough to explain this contrast. The measurement of the first and second order correlation functions yields unambiguous separation between the major decoherence mechanisms and leads to the conclusion that which-way information given by atomic motion is the main source of distinguishability.

\section{B. Parity measurements}

To characterize the maximally entangled state $\left|\Psi^{+}\right\rangle$, we perform a Ramsey interference process that probes the coherence between the $|g e\rangle$ and $|e g\rangle$ states. In our experiment this is realized by a measurement of the parity operator $\hat{P}$ in different bases.

The general state of our two qubit system can be described by the $4 \times 4$ hermitian matrix

$$
\hat{\rho}=\left(\begin{array}{cccc}
\rho_{g g} & \rho_{g g, e g} & \rho_{g g, g e} & \rho_{g g, e e} \\
\rho_{g g, e g}^{*} & \rho_{e g} & \rho_{e g, g e} & \rho_{e g, e e} \\
\rho_{g, g e}^{*} & \rho_{e g, g e}^{*} & \rho_{g e} & \rho_{g e, e e} \\
\rho_{g g, e e}^{*} & \rho_{e g, e e}^{*} & \rho_{g e, e e}^{*} & \rho_{e e}^{*}
\end{array}\right)
$$

Measurement of the parity operator $\hat{P}$ on state $\hat{\rho}$ preceded by the collective rotation $\hat{R}(\theta, \phi)$ can then be formally written as $\operatorname{Tr}\left[\hat{P} \hat{R}(\theta, \phi) \hat{\rho}(\hat{R}(\theta, \phi))^{+}\right]$.

For the $\left|\Psi^{+}\right\rangle$state, it can be readily shown that

$$
\operatorname{Tr}\left(\hat{P} \hat{R}(\pi / 2, \phi)\left|\Psi^{+}\right\rangle\left\langle\Psi^{+}\right|(\hat{R}(\pi / 2, \phi))^{+}\right)=1
$$

for all $\phi$. Parity measurement on the $\left|\Psi^{+}\right\rangle$entangled state is therefore invariant with respect to the change of the rotation pulse $\hat{R}(\pi / 2, \phi)$ phase $\phi$. In order to measure the parity oscillations for this state, we first have to a)

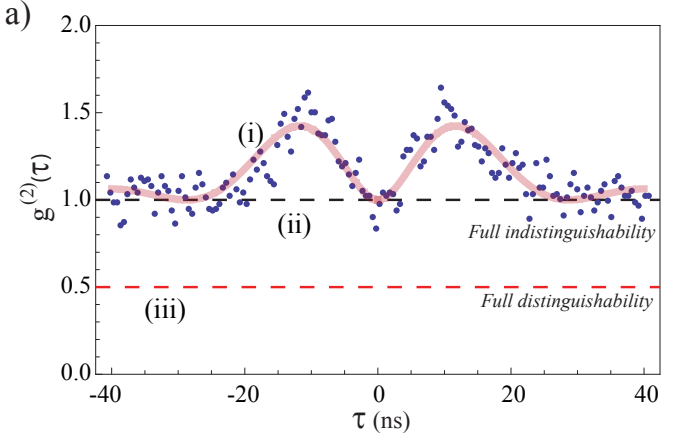

b)

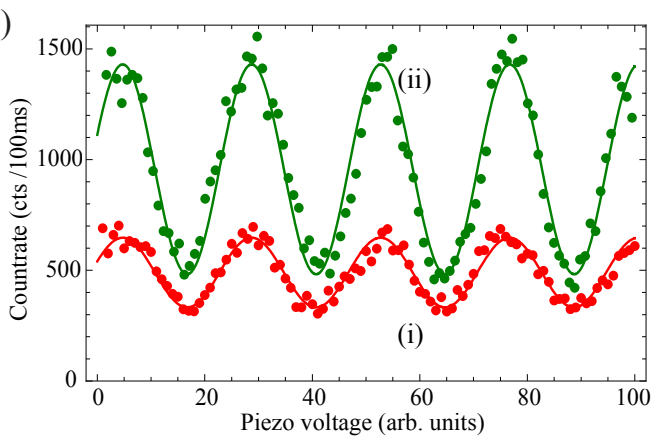

FIG. 3: Correlation functions measurements. a) Second order correlation function of the two ions. Trace (i) shows the experimental results and theoretical fit. Trace (ii) and (iii) are the expected values at $\tau=0$ for fully indistinguishable/distinguishable emitters, respectively. b) Interference fringes when the fluorescence of one ion overlaps with itself (trace (ii)) and when the fluorescence light of both ions is superimposed (trace (i)), in the limit of a weak excitation, as a function of the phase difference between the two optical paths. The contrasts are $60 \%$ and $33 \%$ respectively, both limited mostly by atomic motion.

rotate by a global $\hat{R}(\pi / 2, \pi / 2)$ pulse, corresponding to a $\hat{\sigma}_{y}$ rotation on both qubits with the pulse area of $\pi / 2$.

It can be shown that a peak-to-peak value of the parity measurement oscillation higher than one with an oscillation period of $\phi=\pi$ on two qubits is a sufficient condition for proving that the measured bi-partite system is entangled [5]. To quantify the amount of entanglement, we evaluate the fidelity $F=\left\langle\Psi^{+}|\rho| \Psi^{+}\right\rangle$with the maximally entangled state $\left|\Psi^{+}\right\rangle$. It reads

$$
F=\frac{1}{2}\left[\rho_{g e}+\rho_{e g}+2 \operatorname{Re}\left(\rho_{e g, g e}\right)\right] .
$$

The fidelity thus depends only on the diagonal populations $\rho_{g e}$ and $\rho_{e g}$ and on the real part of the off-diagonal term $\rho_{\text {eg,ge }}$ that expresses the mutual coherence between them. All these terms can be accessed using the collective rotations $\hat{R}$ followed by the parity operator measurement. Diagonal terms $\rho_{g e}$ and $\rho_{e g}$ can be estimated directly by measuring the populations without any prior RF-pulse application. The coherence term $\rho_{e g, g e}$ however cannot be measured with a single global pulse sequence, because 


\begin{tabular}{|c|c|c|}
\hline $\begin{array}{c}\text { Number } \\
\text { of pulses }\end{array}$ & $\begin{array}{c}\text { Pulse } \\
\text { sequence }\end{array}$ & $\begin{array}{c}\text { Measurement } \\
\text { result }\end{array}$ \\
\hline \hline 0 & - & $\rho_{g g}+\rho_{e e}-\left(\rho_{e g}+\rho_{g e}\right)$ \\
\hline \multirow{2}{*}{1} & $\hat{R}(\pi / 2,0)$ & $2 \operatorname{Re}\left(\rho_{\mathrm{ge}, \mathrm{eg}}-\rho_{\mathrm{gg}, \mathrm{ee}}\right)$ \\
& $\hat{R}(\pi / 2, \pi / 4)$ & $2\left(\operatorname{Re}\left(\rho_{\mathrm{ge}, \mathrm{eg}}\right)+\operatorname{Im}\left(\rho_{\mathrm{gg}, \mathrm{ee}}\right)\right)$ \\
& $\hat{R}(\pi / 2, \pi / 2)$ & $2 \operatorname{Re}\left(\rho_{\mathrm{ge}, \mathrm{eg}}+\rho_{\mathrm{gg}, \mathrm{ee}}\right)$ \\
\hline \multirow{2}{*}{2} & $\hat{R}(\pi / 2,0) \hat{R}(\pi / 2, \pi / 2)$ & $2 \operatorname{Re}\left(\rho_{\mathrm{ge}, \mathrm{eg}}-\rho_{\mathrm{gg}, \mathrm{ee}}\right)$ \\
& $\hat{R}(\pi / 2, \pi / 2) \hat{R}(\pi / 2, \pi / 2)$ & $\rho_{g g}+\rho_{e e}-\left(\rho_{\text {eg }}+\rho_{g e}\right)$ \\
\hline
\end{tabular}

TABLE I: Examples of the measurement sequences. Expectation value of the parity operator after applying various global RF-pulse sequences to the ions.

it is always measured together with the coherence term $\rho_{g g, e e}$. A simple way to separate their respective contributions to the measured parity signal is to measure the parity operator value after a single rotation $\hat{R}(\pi / 2, \phi)$ for different phases $\phi$. Independence of the measured parity value on the phase $\phi$ proves that the only coherence term contributing to the measured coherence signal is the desired $\rho_{\text {eg,ge }}$. Table $\Pi$ shows examples of some relevant RFpulses rotation sequences and the corresponding parts of the density matrix $\rho$ contributing to the measured signal.

\section{Quantum coherence of the generated state}

The fidelity of the maximally entangled state $\left|\Psi^{+}\right\rangle$ with the experimentally generated one, is given by [ 6 ]

$$
F=\frac{1}{2} \kappa\left(1+F_{\mathrm{dyn}} e^{-4 t / \tau}\right)
$$

Here $\kappa$ is a factor taking into account imperfect populations of the $|g e\rangle$ and $|e g\rangle$ states mimicked mostly by the detector dark-counts and double-excitations of ions caused by imperfect setting of the Raman-beam polarization and finite value of the excitation probability $p_{e}$. The latter gives a double-excitation rate of $3 p_{e}^{2} \approx 1.5 \times 10^{-2}$. $F_{\text {dyn }}$ describes the decoherence due to atomic motion (dynamical fidelity factor) and $e^{-4 t / \tau}$ expresses the loss of coherence due to the finite coherence time $\tau$ of each individual qubit [7]. Here, $t$ corresponds to the time relevant for this decoherence process, in our case this is the time after the first pulse rotates the generated state out of the decoherence free subspace to the analyzing pulse. The overall fidelity $F$ is related to the respective density matrix elements as defined in (4), through

$$
\begin{array}{r}
\rho_{g e}+\rho_{e g}=\kappa, \\
2 \operatorname{Re}\left(\rho_{e g, g e}\right)=\kappa F_{\mathrm{dyn}} e^{-4 t / \tau} .
\end{array}
$$

The main factor contributing to decoherence is atomic motion. One can show that

$$
F_{\mathrm{dyn}}=e^{\left\langle\left[\vec{q}_{1} \cdot \vec{u}_{1}-\vec{q}_{2} \cdot \vec{u}_{2}\right]^{2}\right\rangle / 2}
$$

where $\vec{q}_{1,2}=\vec{k}_{\text {out }}^{1,2}-\vec{k}_{\text {in }}$ and $\vec{k}_{\text {out }}^{1} \approx-\vec{k}_{\text {out }}^{2} \cdot \vec{k}_{\text {out,in }}^{1,2}$ is the wavevector of the $493 \mathrm{~nm}$ light driving (in) and emitted (out) by atom 1 and 2 respectively. $\vec{u}_{1,2}=\vec{R}_{1,2}-\vec{R}_{1,2}^{O}$ are the displacements of atom 1 and 2 away from their equilibrium positions $\vec{R}_{1,2}^{O}$. Decomposing the ion crystal motion into the normal modes, one gets

$\overrightarrow{q_{1}} \cdot \overrightarrow{u_{1}}-\overrightarrow{q_{2}} \cdot \overrightarrow{u_{2}}=-2 k_{\text {out }} \hat{r}_{\text {cm }}^{\mathrm{rad}}+2 k_{\text {in }} \cos \phi \hat{r}_{\text {rel }}^{\mathrm{rad}}-2 k_{\text {in }} \sin \phi \hat{r}_{\text {rel }}^{\mathrm{ax}}$

where $\hat{r}_{\mathrm{cm}, \mathrm{rel}}^{\mathrm{rad} \text { ax }}$ are the position operators of the quantized harmonic oscillator modes of the two-ion crystal. cm and rel denote the center of mass and stretch/rocking modes, respectively, and rad, ax are the radial and axial coupled modes. $\phi$ is the angle (in our case $\pi / 4$ ) between the Raman excitation laser and the two-ion crystal axis. Inserting the expression 8 into Eq.74, we then get

$$
F_{\text {dyn }}=e^{-2(k \sigma)^{2}},
$$

where

$$
\sigma=\sqrt{\left(\sigma_{\mathrm{cm}}^{\mathrm{rad}}\right)^{2}+\frac{1}{2}\left(\sigma_{\mathrm{rel}}^{\mathrm{rad}}\right)^{2}+\frac{1}{2}\left(\sigma_{\mathrm{rel}}^{\mathrm{ax}}\right)^{2}} .
$$

Each $\sigma$ corresponds to the mean atomic wave packet extent. For instance, we have $\sigma_{\mathrm{cm}}^{\mathrm{rad}}=$ $\sqrt{\left(2 \bar{n}_{\mathrm{cm}}^{\mathrm{rad}}+1\right)\left\langle 0\left|\left(r_{\mathrm{cm}}^{\mathrm{rad}}\right)^{2}\right| 0\right\rangle}$, here $\left\langle 0\left|\left(r_{\mathrm{cm}}^{\mathrm{rad}}\right)^{2}\right| 0\right\rangle$ is the mean extension $\sqrt{\hbar /\left(2 m \omega_{\mathrm{cm}}^{\mathrm{rad}}\right)}$ of the coupled harmonic oscillators in the ground state, and $\bar{n}$ is the mean phonon number in a given mode. $m$ is the atomic mass, and $\omega$ the frequency of the oscillator, which we estimated for all modes using the spectroscopy on the quadrupolar transition to be $\left(\omega_{\mathrm{cm}}^{\mathrm{rad}}, \omega_{\mathrm{rel}}^{\mathrm{ax}}, \omega_{\mathrm{rel}}^{\mathrm{rad}}\right)=2 \pi(1.5,0.9,1.1) \mathrm{MHz}$. Taking the mean phonon number of each mode to be around 12 for a Doppler cooled ion-crystal [4], we get

$$
F_{\text {dyn }}=0.45 \text {. }
$$

In the limit of weak excitation, $\kappa F_{\text {dyn }}$ also directly corresponds to the visibility of the two-ion interference. The effect of motion-induced decoherence can be reduced by cooling the radial modes to the motional ground state [4] or by choosing a forward Raman scattering scenario [6]. The difficulty of the last option is that light from the Raman excitation can leak through the detection channel during the excitation.

The effect of the finite coherence times of the individual qubits is included in the coherence factor $e^{-4 t / \tau}$. In our experiment, the coherence of the individual RF qubits is limited mostly by the ambient magnetic field fluctuations. For each atom, we measured it to be $120 \mu \mathrm{s}$. The noise seen by both ions is correlated when they leave the decoherence free subspace [7]. For our experiment, this amounts to a decrease of our coherence on average to about $e^{-4 t / \tau}=0.96$.

Last, the coherence and overall fidelity of the generated entangled state is limited by the imperfect populations of the desired $|g e\rangle$ and $|e g\rangle$ states. This is effectively 
accounted for in the overall fidelity by the factor $\kappa$, which we estimated from the populations measurements to be $0.89 \pm 0.03$. This is in good agreement with the excitation probability $p_{\mathrm{e}}=0.07 \pm 0.03$ of each ion and the measured dark-counts of our avalanche photodiode of 10 counts/s.

By inserting all the mentioned inefficiencies into the Eq. 4. we get the overall fidelity of our measured state with the maximally entangled state $\left|\Psi^{+}\right\rangle$to be $F=0.62$, in good agreement with the measured fidelity of $0.64 \pm$ 0.02 .

\section{Measurement of the second-order correlation function}

In order to estimate the degree of the spatial and polarization indistinguishability of the photons coming from the two ions, we measure the second order correlation function $g^{(2)}(\tau)$. To reach a time-resolution beyond the spontaneous decay time, we implemented a Hanbury Brown and Twiss set-up, by splitting the fluorescence into two parts with a non-polarizing beam-splitter and inserting a second avalanche photodiode (APD2). The unnormalised correlation function $G^{(2)}(\tau)$ reads

$$
G_{\mathrm{Tot}}^{(2)}(\tau)=\left\langle\hat{E}^{-}(t) \hat{E}^{-}(t-\tau) \hat{E}^{+}(t-\tau) \hat{E}^{+}(t)\right\rangle
$$

where $\hat{E}^{+}$and $\hat{E}^{-}$are positive and negative frequency parts of the field operator $\hat{E}$ and $\tau$ is the time delay between two clicks at the two detectors. Field $\hat{E}=$ $\vec{e}_{1} \hat{E}_{1}+\vec{e}_{2} \hat{E}_{2} e^{i \phi}$ corresponds to the coherent sum of the two field operators from atoms 1 and 2 with a phase difference $\phi$ and $\vec{e}_{1}$ and $\vec{e}_{2}$ are the polarization vectors. For this measurement, we do not stabilize the optical paths. By inserting the field expression into equation (12), and averaging over the optical phases $\phi$, we get $G_{\text {Tot }}^{(2)}(\tau)=2\left(G^{(2)}(\tau)+\left|\vec{e}_{1} \vec{e}_{2}\right|^{2}\left|G^{(1)}(\tau)\right|^{2}+\langle n\rangle^{2}\right)$, where $G^{(1)}(\tau)$ is the (unnormalised) single ion first order correlation function $G^{(1)}(\tau)=\left\langle\hat{E}^{-}(t) \hat{E}^{+}(t+\tau)\right\rangle$ and $\langle n\rangle$ is the mean number of photons on each APD.

When normalizing the second order correlation to
$\left\langle\hat{E}^{-}(t) \hat{E}^{+}(t)\right\rangle^{2}$, one obtains

$$
g_{\text {Tot }}^{(2)}(\tau)=\frac{1}{2}\left(g^{(2)}(\tau)+\left|\overrightarrow{e_{1}} \overrightarrow{e_{2}}\right|^{2}\left|g^{(1)}(\tau)\right|^{2}+1\right)
$$

where $g^{(1)}$ and $g^{(2)}$ stand for first and second order normalized correlation functions, respectively. For two single atoms, $g^{(2)}(0)=0$ (antibunching) and $g^{(1)}(0)=1$, so that total second order correlation function is equal to $g_{\text {Tot }}^{(2)}(0)=\frac{1}{2}\left(1+\left|\overrightarrow{e_{1}} \overrightarrow{e_{2}}\right|^{2}\right)$. From here it follows, that for two indistinguishable polarizations $g_{\text {Tot }}^{(2)}(0)=1$. The same analysis applies also for the spatial indistinguishability. In our experiment $g_{\text {Tot }}^{(2)}(0)=0.98 \pm 0.07$, which shows that indistinguishability conditions are fulfilled and that temporal decoherence in the form of atomic motion is the main limitation.

\section{E. Efficiency budget}

The efficiency for detecting a single Raman-scattered photon in our setup was estimated to be $\eta=8 \times 10^{-4}$. It was derived from the detection probability of a single Raman scattered photon given by the collection efficiency of our lenses $(\sim 0.04)$, the single-mode fiber coupling efficiency $(\sim 0.1)$ and by the avalanche-photodiode detection efficiency $(\sim 0.6)$. Additional factors of 0.5 and 0.66 come from the polarization filtering of the unwanted $\pi$-polarized photons and from the probability of decaying back to the $|g\rangle$ state after the Raman pulse excitation, respectively. The actual entanglement rate may thus be estimated. With our single ion excitation probability $p_{e}=0.07 \pm 0.03 \%$, the overall probability for detecting a single photon from one of the two ions is then $P_{\text {succ }} \sim 2 p_{e} \eta=1.1 \times 10^{-4}$. For comparison, the heralding entanglement scheme proposed by Simon et al. 8 would give for our experimental setup approximately $P_{\text {succ }} \approx 2 \eta^{2}=1.3 \times 10^{-6}$, so about two orders of magnitude smaller success probability of entanglement generation in a given experimental trial. For simplicity, we assumed here $p_{e}=1$ for the two-photon scheme and an additional factor of two comes from the two possible contributions to the coincidence detection events.
[1] Eichmann, U. et al. Phys. Rev. Lett. 70, 220402 (2004).

[2] Itano, W. M. et al. Phys. Rev. A 57, 4176-4187 (1998).

[3] Eschner, J., Raab, C., Schmidt-Kaler, F. \& Blatt, R. Nature 413, 495-498 (2001).

[4] Slodička, L. et al. Phys. Rev. A 85, 043401 (2012).

[5] Sackett, C. A. et al. Nature 404, 256-258 (2000).
[6] Cabrillo, C., Cirac, J. I., Garcia-Fernández, P. \& Zoller, P. Phys. Rev. A 59, 1025-1033 (1999).

[7] Monz, T. et al. Phys. Rev. Lett. 106, 130506 (2011).

[8] Simon, C. \& Irvine, W. T. M. Phys. Rev. Lett. 91, 110405 (2003). 